\title{
Optimizing the management of patients with small renal masses in a Canadian context: A Markov decision-analysis model
}

\author{
Kristen McAlpine, MD, FRCSC ${ }^{*}$; Maneesh Sud, MD, FRCPC ${ }^{* 2}$; Antonio Finelli, MD, MSc, FRCSC ; \\ Girish S. Kulkarni, MD, PhD, FRCSC'
}

'Division of Urology, Department of Surgery, Princess Margaret Cancer Centre, University Health Network and the University of Toronto, Toronto, ON, Canada; ${ }^{2}$ Schulich Heart Program, Division of Cardiology, Sunnybrook Health Sciences Centre, University of Toronto, Toronto, ON, Canada

*Equal contributors

Cite as: McAlpine K, Sud M, Finelli A, et al. Optimizing the management of patients with small renal masses in a Canadian context: A Markov decision-analysis model. Can Urol Assoc J 2022;16(1): E32-8. http://dx.doi.org/10.5489/cuaj.7301

Published online August 26, 2021

Appendix available at cuaj.ca

\section{Abstract}

Introduction: The management of patients with a small renal mass (SRM) varies significantly. The objective of this study was to determine which initial management strategy resulted in the greatest quality-adjusted life months (QALM) for an index patient with a SRM.

Methods: A Markov decision analysis was used to determine the effect of 1) treating patients with a partial nephrectomy (PN); 2) active surveillance (AS); and 3) renal mass biopsy on QALM over a 10-year horizon. All relevant health states were modelled. Biopsy sensitivity and specificity were modelled assuming an $80 \%$ prevalence of cancer using procedural pathology as the gold standard. Health state utilities were obtained from the Tufts Medical Centre Cost-Effective Analysis Registry. Deterministic sensitivity analyses were used to test key assumptions.

Results: Over a 10-year time horizon for a 70-year-old male with a $2 \mathrm{~cm}$ SRM, the biopsy strategy resulted in 38.07 QALM, whereas treating all patients with PN resulted in 37.69 QALM and AS in 36.25 QALM. The model was most sensitive to the probability that a patient would remain alive at baseline. Biopsy was the preferred strategy when sensitivity was greater than $77 \%$. As the underlying probability of cancer increased, the threshold of renal mass biopsy sensitivity to still favor biopsy increased.

Conclusions: Renal mass biopsy is the preferred initial management strategy for an index patient with a SRM to optimize QALM. When the probability of cancer is high, centers should aim for a sensitivity of at least $77 \%$ in order to consider a biopsy as the first strategy.

\section{Introduction}

The incidence of kidney cancer has increased steadily over the last 30 years. ${ }^{1,2}$ With the increased availability and use of ultrasounds and cross-sectional abdominal imaging, the majority of kidney cancers are now diagnosed incidentally as small renal masses (SRM). ${ }^{2,3}$ A SRM is typically defined as a tumor in the kidney that measures $<4 \mathrm{~cm} .{ }^{4}$ The majority of these tumors are cancerous, however, approximately $20 \%$ of SRM are benign..$^{5,6}$ Interestingly, despite this global trend of diagnosing kidney cancers at an earlier stage, the mortality rate of kidney cancer has not changed significantly over this same time frame. ${ }^{2}$ This raises questions about whether we are over-diagnosing and over-treating many patients who would never be impacted by the SRM over the course of their lifetime. The concept of over-diagnosis and over-treatment is most pronounced for older patients with multiple comorbidities. These patients have a higher risk of death from competing causes and are at increased risk of complications from treatment of the SRM. ${ }^{7}$ Thus, there may be a greater risk of harming these patients than helping them by treating a SRM with an invasive procedure. ${ }^{8}$

The management of patients with a SRM varies significantly. Historically, radical surgery to remove the entire kidney would have been recommended. ${ }^{9,10}$ In contemporary times, management options include: 1) surgery to remove the tumor with a partial nephrectomy (PN); 2) thermal ablation of the tumor (if anatomically possible); and 3) active surveillance (AS) to monitor the tumor with regular imaging., ${ }^{4,11}$ To inform the decision between surgery, ablation, and AS, a renal mass biopsy can be used to determine the pathology of a SRM. Benchmarks for the sensitivity and specificity of renal mass biopsy have not been defined, however, and it is possible that poorly performed biopsies may expose patients to additional harm without benefit. In the development of the recent Kidney Cancer Research Network of Canada's (KCRNC) best practice report on biopsy for renal masses, there was a fair amount of discussion regarding the limitations of benchmarking the parameters for renal mass biopsies across Canada because the majority of the large studies had been done in centers of excellence. ${ }^{12}$ This group of kidney cancer experts called for an assessment of 
the realistic benchmarks that small centers should aim for to ensure they are meeting standards of care. ${ }^{12}$ Given the complexity of competing risks for these patients, utilities and disutilities of each disease state, and characteristics of renal mass biopsy, decision analysis modelling allows for assessment of these questions and sensitivity analyses to compare ranges of probabilities for each variable.

The objective of this study was to determine which of renal mass biopsy, $\mathrm{PN}$, or AS resulted in the greatest number of quality-adjusted life months (QALM). A secondary objective was to assess the minimum sensitivity and specificity required for renal mass biopsy to produce the greatest QALM relative to $\mathrm{PN}$ and $\mathrm{AS}$ at academic and community centers across Canada.

\section{Methods}

\section{Model structure}

A decision tree was constructed for patients with a SRM (Appendix 1; available at cuaj.ca). Three management strategies were modeled, including: 1 ) treating all patients with $\mathrm{PN}$; 2) starting all patients on AS; or 3) proceeding first with renal mass biopsy. Thermal ablation was not included in this model, as it is not available at all centers in Canada and not all SRMs are amenable to ablation due to their size and location within the kidney. In each management pathway, the underlying probability that the SRM was cancerous was incorporated. Following completion of the initial treatment pathway in which patients received a $\mathrm{PN}$, renal mass biopsy, or were started on AS, they entered one of four Markov health-state transition models with a cycle length of one month and a time horizon of 10 years. The outcome of interest was QALM. This outcome was chosen since invasive and conservative strategies may drastically effect health utilities without significantly increasing quantity of life in these patients. The base case was set as a 70-year-old healthy male patient with a $2 \mathrm{~cm}$ renal tumor who would be willing and able to receive any of the management strategies. This base case is reflective of the index patient reported in large series of patients managed for SRMs. ${ }^{13-17}$ Half-cycle correction was employed for utility calculations, with standard discounting rates of $1.5 \%$. All modelling was performed on TreeAge Pro, Healthcare edition. ${ }^{18}$

\section{Treatment strategies}

\section{Treat all patients with partial nephrectomy}

In this management arm, all patients received a PN. This model assumes that the tumor was amendable to a PN and that an open approach to the surgery was used. Ablative therapies were not included in the model, as they are not widely available in all centers. Adverse postoperative events were included in the model with their associated disutilities. After $\mathrm{PN}$, patients entered separate Markov models depending on the underlying probability the SRM was cancerous. For noncancerous lesions, patients entered a Markov model in which they had the probability of dying or living per cycle based on a healthy age-matched population. A state-transition diagram for this Markov model (M0) is shown in Appendix 2 (available at cuaj.ca). These patients still received the disutility associated with surgery. Patients with a cancerous tumor entered a separate Markov model (M1) in which they all began in a "post-PN with no cancer" disease state. These patients could remain in this state or could then cycle into other disease states, including "local recurrence," "distant metastases," or "death." It was assumed that patients who developed a local recurrence would proceed with a radical nephrectomy. Patients who received a radical nephrectomy could then enter a "post-radical nephrectomy" state or could enter "distant metastases" or "death." Adverse postoperative events associated with radical nephrectomy were modelled as transition states in the Markov model.

\section{Active surveillance}

In this management arm, patients entered one of two Markov models based on their probability of having a cancerous SRM. All patients with a cancerous SRM entered the Markov model in the "no local progression" disease state (M2). These patients could remain in the "no local progression" state or could cycle to other disease states, including "local progression," "metastatic disease," or "death." It was assumed that a patient who developed local progression would proceed with a PN. Patients who received a PN could then enter the "post-PN," "metastatic disease," or "death" states. Patients who received a PN could develop "local recurrence" and it was assumed that these patients would then receive a radical nephrectomy and would enter a "post-radical nephrectomy" state, as described above. Patients could remain in the "post-radical nephrectomy" state or could cycle into "distant metastases" or "death" states. Finally, patients with benign SRM entered a similar Markov model, where they were able to develop local progression triggering a PN; however, these patients would not have the possibility of developing local recurrence or distant metastatic disease based on their noncancerous tumor (M3).

\section{Renal mass biopsy}

Patients entering the renal mass biopsy arm received a biopsy with the possibility of a complication after biopsy. The biopsy had the potential to be diagnostic or non-diagnostic. A diagnostic biopsy indicated that the tumor was appropriately sampled, and the tissue obtained was adequate for pathological review. A non-diagnostic biopsy indicated that the tumor was missed, and a diagnosis was not possible based 
on the tissue obtained. It was assumed that a patient who received a non-diagnostic first renal mass biopsy would proceed with a second attempt, once again with the possibility of a complication. If this second attempt at a biopsy was again non-diagnostic, it was assumed that these patients would proceed with $\mathrm{PN}$, as described above. A summary of all assumptions in the model is available in Appendix 3 (available at cuaj.ca).

For patients who received a diagnostic biopsy, the sensitivity and specificity of renal mass biopsy were modelled. It was assumed that all patients with cancerous pathology on biopsy would proceed to surgery: all patients who received a "true-positive" or "false-positive" diagnosis of cancer proceeded to surgery in the form of a PN, as described above. Following PN, patients with a true-positive biopsy entered a Markov model in which local recurrence and metastatic disease remained possible (M1). However, patients with a false-positive biopsy were not at risk for metastatic disease or local recurrence after PN, given the non-cancerous tumor, and entered a separate Markov model. It was assumed that all patients who had a diagnostic biopsy that returned with a non-cancerous pathology would proceed to AS. Patients who received a "true-negative" diagnosis of "no cancer" on biopsy entered into an AS Markov model for patients with benign SRM (M3). Patients who received a "false-negative" diagnoses of "no cancer" on renal mass biopsy, entered into an AS Markov model for patient with a cancerous SRM (M2).

\section{Model probabilities and utilities}

A review of the literature was performed to assess the available data for each of the variables. Summary of evidence tables were created (Appendix 4; available at cuaj.ca). The highest quality of evidence available to inform each probability was used. The prevalence of cancer for the base case and the range of possible prevalence of cancer for other patients were determined. Utilities and disutilities were obtained from the Tufts Medical Centre Cost-Effective Analysis Registry. ${ }^{19}$ The utilities and disutilities used in the model are shown in Table 1. When no available utility existed for a given variable, the best available utility from a similar population was used. Statistics Canada life tables were used to inform a patient's probability of remaining alive for each one-month cycle based on an aged-matched population. ${ }^{20}$

\section{Statistical analysis}

The primary analysis of interest was to assess which of the management arms resulted in the greatest number of QALM over a 10-year time horizon. A 10-year time horizon was the primary analysis used due to the lack of studies reporting long-term data to inform the probabilities in the model beyond 10 years. The model was also run over a lifetime horizon for comparison. The base case characteristics and key transition probabilities were validated against the opinion of an expert in the field (Table 2). Deterministic one-way and two-way sensitivity analyses were planned to assess the effect of each variable on the preferred management strategy. Two-way sensitivity analyses of greatest clinical interest were those that assessed the effect of each variable on the preferred management strategy while varying the prevalence of cancer. In keeping with the secondary objective for this study, the impact of sensitivity and specificity of renal mass biopsy on the preferred strategy while adjusting the prevalence of cancer was determined. Internal validation was done by both lead authors (KM, MS) who independently examined the model, conducted one-way sensitivity analyses to ensure results were clinically plausible, and verified calculations performed in TreeAge.

\begin{tabular}{|c|c|c|c|}
\hline State & Utility in model & $\begin{array}{l}\text { Range of utilities in } \\
\text { registry }\end{array}$ & Reference \\
\hline Alive post-partial nephrectomy & 0.75 & $0.744-0.755$ & Patel, Abdo Radiol 2020 \\
\hline Alive, on active surveillance & 0.81 (prostate cancer) & $0.81-0.99$ & White, Cancer 2019 \\
\hline Metastatic cancer & 0.54 & $0.25-0.81$ & Wu, PLoS One 2011 \\
\hline $\begin{array}{l}\text { Alive with recurrence post-partial } \\
\text { nephrectomy }\end{array}$ & 0.73 (breast cancer) & $0.4-0.76$ & Wei, Clin Drug Investig 2019 \\
\hline State & Disutility & $\begin{array}{l}\text { Range of disutilities } \\
\text { in registry }\end{array}$ & Reference \\
\hline Biopsy & -0.006 & -0.5 to -0.003 & Barnett, BJUI 2016 \\
\hline
\end{tabular}




\begin{tabular}{lcc}
\hline \multicolumn{3}{l}{$\begin{array}{l}\text { Table 2. Validation of probabilities and base case } \\
\text { characteristics used in model }\end{array}$} \\
\hline Variable & Value in model & Expert opinion \\
\hline $\begin{array}{l}\text { Rate of metastatic disease on } \\
\text { active surveillance }\end{array}$ & $2 \%$ over 5 years & $2 \%$ at 5 years \\
$\begin{array}{l}\text { Rate of progression on active } \\
\text { surveillance }\end{array}$ & $\begin{array}{c}25 \% \text { over } 5 \\
\text { years }\end{array}$ \\
$\begin{array}{l}\text { Rate of complication on biopsy } \\
\text { Rate of partial nephrectomy }\end{array}$ & $0.7 \%$ & $25 \%$ \\
complication & $10 \%$ & $<1 \%$ \\
Base case & & $10-15 \%$ \\
& 70 years old & 65 years old \\
& Male & Male \\
& $2 \mathrm{~cm}$ tumor & $3 \mathrm{~cm}$ tumor \\
\hline
\end{tabular}

\section{Results}

The preferred strategy for the base case at a 10-year horizon was renal mass biopsy, which offered 38.07 QALM (Table 3). This was followed by the PN strategy at 37.69 QALM. AS was the least preferred strategy, resulting in 36.25 QALM. These results were stable with and without the discount rate applied (Table 3). When the analysis was extended to a lifetime horizon, the results were similar, with renal mass biopsy remaining the preferred strategy at 60.71 QALM, followed by surgery at 60.29 QALM, and AS at 50.64 QALM.

\section{One-way sensitivity analyses}

The model was found to be internally valid and free of errors through one-way sensitivity analyses of each variable performed by both lead authors. One-way sensitivity analyses of each of the variables included in the model indicated that the results of the model were most impacted by the probability an individual would remain alive over the course of a cycle in the Markov models and by the utility placed on the post-PN disease state (Fig. 1). Other important variables that were highly impactful on the preferred strategy were the prevalence of cancer and the discount rate.

Several variables had thresholds at which the preferred strategy changed. For example, for the variable "probability

\begin{tabular}{lcc}
\hline \multicolumn{3}{l}{ Table 3. Results of model for 10-year and lifetime horizon } \\
\hline Strategy & $\begin{array}{c}\text { QALM } \\
\text { (discounted) }\end{array}$ & $\begin{array}{c}\text { QALM } \\
\text { (undiscounted) }\end{array}$ \\
\hline $\begin{array}{l}\text { 10-year horizon } \\
\text { Treat all }\end{array}$ & 37.69 & 40.42 \\
Biopsy & 38.07 & 40.81 \\
Active surveillance & 36.25 & 38.75 \\
Lifetime horizon & & \\
Treat all & 60.29 & 69.06 \\
Biopsy & 60.71 & 69.52 \\
Active surveillance & 50.64 & 64.27 \\
\hline OALM: quality adjusted life months. & & \\
\hline
\end{tabular}

of dying in the operating room," if the probability was less than 0.06, the preferred strategy was renal mass biopsy; however, if the probability was greater than 0.06 , the preferred strategy was AS. The thresholds that were detected for various variables in the model are displayed in Table 3.

\section{Two-way sensitivity analyses}

Two-way sensitivity analyses assessing the impact of renal mass biopsy characteristics and the prevalence of cancer on the preferred strategy are shown in Figs. 2 and 3. When the prevalence of cancer was high, then a highly sensitive renal mass biopsy resulted in biopsy being the preferred strategy over upfront $\mathrm{PN}$. When the prevalence of cancer was low, a less sensitive renal mass biopsy was permitted to allow biopsy to remain the preferred strategy over PN. In Fig. 2, the $x$-axis shows the sensitivity of biopsy ranging from $50-100 \%$, and the $y$-axis shows the prevalence of cancer ranging from $40-100 \%$. Using Fig. 2, a patient population's baseline risk of malignancy in the SRM can be identified on the $y$-axis and then a tangential line can be drawn where the preferred strategy changes in order to determine the sensitivity of biopsy required to impact the preferred strategy for that group of patients. For example, for the base case with a baseline prevalence of cancer of $80 \%$ (based on demographic and tumor characteristics), the sensitivity

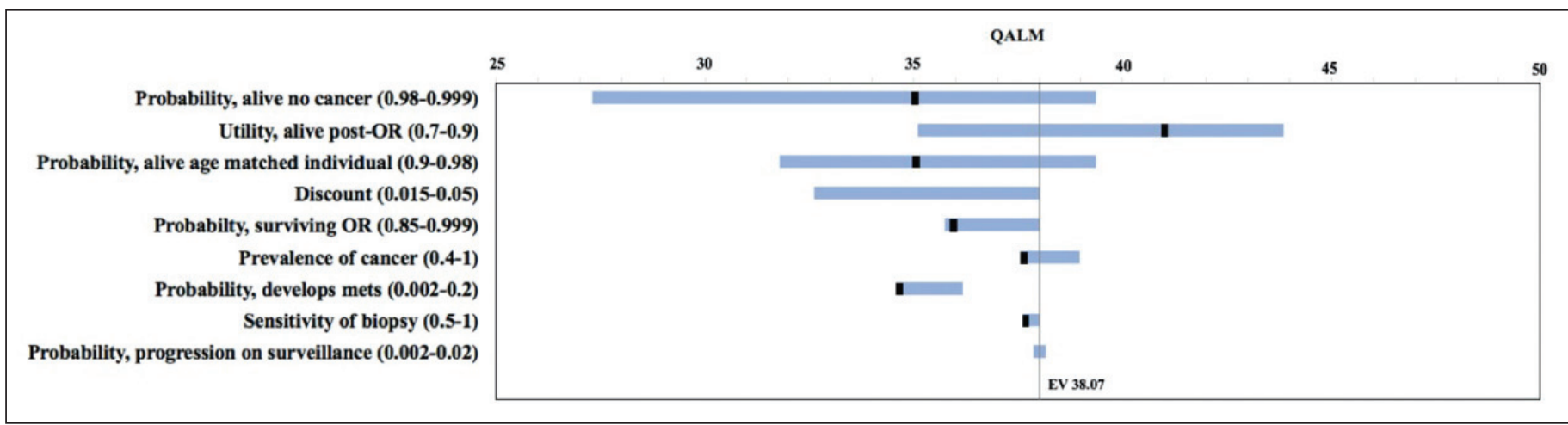

Fig. 1. Tornado plot for results of one-way sensitivity analyses for highly impactful variables. EV: expected value; mets: metastases; OR: operation; QALM: qualityadjusted life months. 
of a biopsy at which the strategy changed from upfront PN to renal mass biopsy was $77 \%$ (Fig. 2).

A second two-way sensitivity analysis assessed the preferred strategy when ranges of specificity of biopsy and prevalence of cancer were assessed. This two-way sensitivity analysis showed that renal mass biopsy was the preferred strategy for the vast majority of patients if the specificity of biopsy ranged from $50-100 \%$ unless the prevalence of cancer was $98 \%$, at which point treating all patients with PN would be preferred. For the base case, biopsy was always the preferred approach when the specificity of the biopsy ranged from $50-100 \%$ (Fig. 3).

\section{Discussion}

In this Markov decision analysis, we found that the preferred strategy for an index patient with a SRM would be to receive a renal mass biopsy prior to deciding on management. In our model, patients who received a renal mass biopsy had the highest QALMs at 38.07 over a 10-year time horizon, which was superior to both proceeding directly to surgery or directly to AS at 37.69 and 36.25 QALMs, respectively.

The contemporary role of renal mass biopsy in the management of patients with a SRM is controversial. Most SRM are cancerous; however, approximately $20 \%$ are benign. ${ }^{5}$ The probability that a SRM is malignant varies by the sex and age of the patient, as well as the size of the tumor. ${ }^{6}$ Many physicians feel confident triaging patients to management strategies based on their baseline health status, the size of the tumor, and the likelihood that the tumor is malignant. However, without a biopsy, patients may be exposed to the

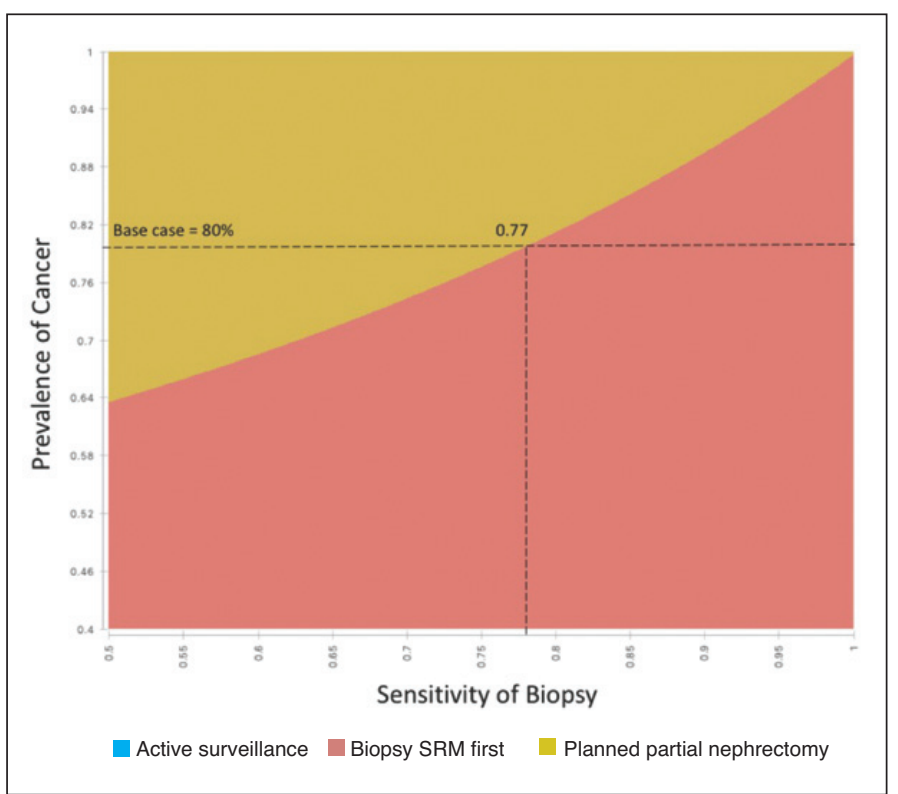

Fig. 2. Two-way sensitivity analysis on sensitivity of biopsy and prevalence of cancer. SRM: small renal mass. risks of surgery for a benign tumor or may miss the window of cure for an aggressive cancer monitored on AS. For this reason, there are some kidney cancer experts who are strong proponents of renal mass biopsy prior to management. The results of this Markov model would indicate that this approach would be preferred for an index patient with a SRM.

Renal mass biopsy is highly reliant on the skillset of the radiologist performing the biopsy and on the pathologist reviewing the tissue obtained from the biopsy. Most of the large series reporting results of renal mass biopsy are from experienced academic centers with highly trained individuals performing and reviewing the renal mass biopsies. ${ }^{21}$ Many smaller centers do not have access to such highly specialized individuals and argue that the high degree of accuracy quoted in these large series lacks external generalizability. In 2019, the KCRNC met to develop a consensus statement on the role of renal mass biopsy for physicians and patients across Canada. ${ }^{12}$ During this meeting, the unknown target for accuracy of renal mass biopsies for each center was highlighted as a significant limitation in the available literature. Questions have also been raised about the centralization of renal mass biopsy to centers of excellence that are able to obtain high levels of sensitivity and specificity while minimizing complications. Our decision analysis model directly answers the questions posed by these kidney cancer experts. In the two-way sensitivity analyses for an index patient with a baseline risk of a cancerous SRM of $80 \%$, the benchmark sensitivity of renal mass biopsy is $77 \%$. The specificity of renal mass biopsy had less of an impact on the preferred strategy when a range of $50-100 \%$ was assessed in a two-way sensitivity analysis with the prevalence of cancer. Thus, centers should aim to optimize the sensitivity of renal mass biopsy to ensure the results are impactful for patients even if improving sensitivity results in a decreased specificity. Centers that are unable to obtain a renal mass biopsy sensitivity rate of at least $77 \%$ should refer patients to center of excellence and/or should invest in training individuals in this skill set to meet this benchmark.

The results of this study are important, as they will inform practice and will establish benchmarks for renal mass biopsy proficiency in centers across Canada. The strengths of this study are its novel analysis, strict adherence to good modelling practices, and its ability to directly affect clinical practice. The model can also be adapted to reflect patient characteristics, tumor characteristics, and hospital biopsy characteristics to properly model the clinical situation.

There are limitations to this study. First, there were several assumptions regarding the serial steps in the model for patients who experienced a given outcome. For example, it was assumed that all patients with a local recurrence after a $\mathrm{PN}$ would proceed to receive a radical nephrectomy. These assumptions may not be accurate for all patients, given the nuances of each patient's unique clinical scenario. Second, 


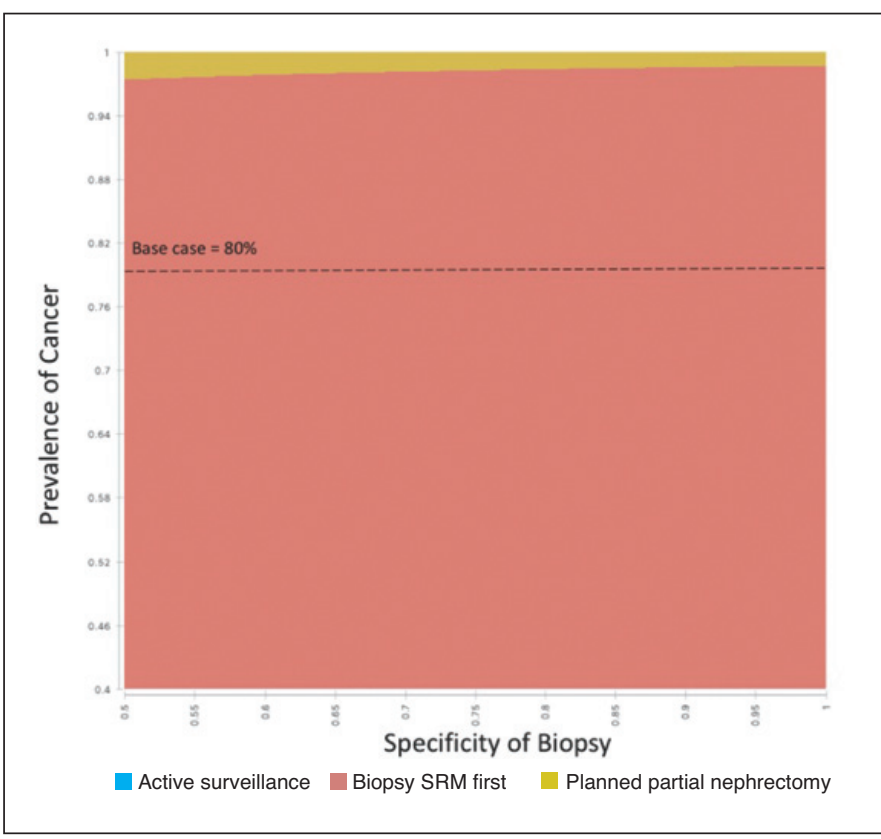

Fig. 3. Two-way sensitivity analysis on specificity of biopsy and prevalence of cancer. SRM: small renal mass.

the model does not include thermal ablation as a possible management option. This was done intentionally, as the model was developed to assess for outcomes in both smaller community hospitals and larger academic hospitals. In many centers outside of tertiary care facilities, thermal ablation is not available to treat SRMs. Additionally, there is limited long-term outcome data for thermal ablative techniques used to treat patients with a SRM. Modelling the preferred strategy using short-term outcomes of ablative techniques compared to longer-term outcomes of surgery and surveillance could have introduced bias into the model. Third, we did not include a cost-utility analysis in this study. Although assessing the cost of each management option would have been beneficial, we felt that it did not directly address the objective of this study. Finally, the literature available on the long-term outcomes for patients managed with a SRM is limited, with sparse data beyond a 10-year time horizon. The primary analysis assessing the preferred strategy to optimize QALM over a 10-year time horizon is likely reasonable in older patients, however, may not be as applicable in younger patients. As more long-term data become available, this model can be updated to allow for more accurate assessment of the impact of a lifetime horizon on the preferred strategy to optimize QALM.

\section{Conclusions}

This Markov decision analysis highlights the importance of renal mass biopsy in the management of patients with a SRM. Based on the results of this study, renal mass biopsy should be more widely incorporated into the management of patients with SRM. By benchmarking the success rate of renal mass biopsy across Canada, we can ensure patients with a SRM receive high-quality care in order to properly inform the decision of their management.

Competing interests: Dr. Finelli has been an advisory board member for Abbvie, Astellas, Bayer, Janssen, Ipsen, Sanofi, and TerSera; and has participated in clinical trials supported by Astellas, Bayer, and Janssen Dr. Kulkarni has been an advisory board member for Astellas, Ferring, Janssen, Merck, Roche, and Theralase; has received honoraria from Abbvie, Ferring, Sanofi, and TerSera; and has participated in clinical studies supported by AstraZeneca, Bristol Myers Squibb, Janssen, Merck, and Theralase. The remaining authors do not report any competing personal or financial interests related to this work.

This paper has been peer-reviewed.

\section{References}

1. Kane CJ, Mallin K, Ritchey J, et al. Renal cell cancer stage migration. Cancer 2008;1 13:78-83. https://doi.org/10.1002/cncr.23518

2. Patel HD, Gupta $M$, Joice $G A$, et al. Clinical stage migration and survival for renal cell carcinoma in the United States. Eur Urol Oncol 2019;2:343-8. https://doi.org/10.1016/i.euo.2018.08.023

3. Volpe A, Panzarella $T$, Rendon RA, et al. The natural history of incidentally detected small renal masses. Cancer 2004; 100:738-45. https://doi.org/10.1002/cncr.20025

4. Jewett MAS, Rendon R, Lacombe L, et al. Canadian guidelines for the management of small renal masses (SRM). Can Urol Assoc J 2015;9:160-3. https://doi.org/10.5489/cuaj.2969

5. Bhindi B, Thompson RH, Lohse CM, et al. The probability of aggressive vs. indolent histology based on renal tumor size: Implications for surveillance and treatment. Eur Urol 2018;74:489-97. https://doi.org/10.1016/i.eururo.2018.06.003

6. Lane B, Babineau D, Kattan M, et al. A preoperative prognostic nomogram for solid enhancing renal tumors $7 \mathrm{~cm}$ or less amenable to partial nephrectomy. J Urol 2007;178:429-34. https://doi.org/10.1016/i. juro.2007.03.106

7. Kutikov A, Egleston B, Canter D, et al. Competing risks of death in patients with localized renal cell carcinoma a comorbidity-based model. J Urol 2015;188:2077-83. https://doi.org/10.1016/i. juro.2012.07.100

8. Sohlberg EM, Metzner TJ, Leppert JT. The harms of overdiagnosis and overtreatment in patients with small renal masses: A mini-review. Eur Urol Focus 2019;5:943-5. https://doi.org/10.1016/i. euf.2019.03.006

9. Volpe A, Cadeddu JA, Cestari A, et al. Contemporary management of small renal masses. Eur Urol 2011;60:501-15. https://doi.org/10.1016/j.eururo.2011.05.044

10. Almassi N, Gill BC, Rini B, et al. Management of the small renal mass. Trans/ Androl Urol 2017;6:923-30. https://doi.org/10.21037/tau.2017.07.11

11. Campbell S, Uzzo RG, Allaf ME, et al. Renal mass and localized renal cancer: AUA guideline. J Urol 2017;198:520-9. https://doi.org/10.1016/i.juro.2017.04.100

12. Lavallée LT, McAlpine K, Kapoor A, et al. Kidney Cancer Research Network of Canada (KCRNC) consensus statement on the role of renal mass biopsy in the management of kidney cancer. Can Urol Assoc J 2019;13:377-83. htrps://doi.org/10.5489/cuaj.6176

13. Smaldone MC, Kutikov A, Egleston BL, et al. Small renal masses progressing to metastases under active surveillance: A systematic review and pooled analysis. Cancer 2012;118:997-1006. https://doi.org/10.1002/cncr.26369

14. Richard PO, Jewett MAS, Tanguay $S$, et al. Safety, reliability, and accuracy of small renal tumour biopsies: Results from a multi-institution registry. BJU Int 2017;119:543-9. https://doi.org/10.1111/bju.13630

15. Nayan $M$, Saarela 0 , Lawson $K$, et al. Long-term outcomes after radical or partial nephrectomy for Tla renal cell carcinoma: A population-based study. Can Urol Assoc J 2020;14392-7. https://doi.org/10.5489/cuaj.6343

16. Uzosike AC, Patel HD, Alam R, et al. Growth kinetics of small renal masses on active surveillance: Variability and results from the DISSRM registry. J Urol 2018;199:641-8. https://doi.org/10.1016/i. juro.2017.09.087 
McAlpine et al

17. Garbens A, Wallis CJD, Klaassen Z, et al. Comprehensive assessment of the morbidity of renal mass biopsy: A population-based assessment of biopsy-related complications. Can Urol Assoc J 2020;15:42-7. https://doi.org/10.5489/cuai.6477

18. TreeAge Pro, Healthcare Edition, Copyright 1988-2020 TreeAge Software, LLC. All rights reserved. 2020.

19. Center for the Evaluation of Value and Risk in Health. The Cost-Effectiveness Analysis Registry. (Boston) Institute for Clinical Research and Health Policy Studies, Tufts Medical Center. Available at: www.cearegistry. org. Accessed Nov. 20, 2020.

20. Statistics Canada. Life Tables, Canada, Provinces and Territories (84-537-X). 2020;(84). Available at: http://www5.statcan.gc.ca/ol-cel/olc.action?obild=84-537-X\&objType=2\&lang=en\&limit=1. Accessed Aug. 26, 2021.
21. Marconi L, Dabestani S, Lam TB, et al. Systematic review and meta-analysis of diagnostic accuracy of percutaneous renal tumour biopsy. Eur Urol 2016;69:660-73. https://doi.org/10.1016/i.euru10.2015.07.072

Correspondence: Dr. Girish Kulkarni, Division of Urology, Department of Surgery, Princess Margaret Cancer Centre, University Health Network and the University of Toronto, Toronto, ON, Canada; girish.kulkarni@uhn.ca 